\title{
Obtenção e caracterização físico-química de petit suisse prebiótico de búfala
}

\author{
Obtaining and physicochemical characterization of prebiotic petit suisse of buffalo \\ Obtención y caracterización física y química de petit suisse prebiotica de búfala
}

Rebeca Morais Silva Santos

ORCID: https://orcid.org/0000-0003-0867-2795

Universidade Federal de Campina Grande, Brasil

E-mail: rebecamoraiscg@gmail.com

Thaisa Abrantes Souza Gusmão

ORCID: https://orcid.org/0000-0001-8640-7036

Universidade Federal de Campina Grande, Brasil

E-mail: ta_brantes@hotmail.com

Rennan Pereira de Gusmão

ORCID: https://orcid.org/0000-0002-7355-8078

Universidade Federal de Campina Grande, Brasil

E-mail: rennangusmao@gmail.com

Ingrid Paloma Conrado Garrido

ORCID: https://orcid.org/0000-0001-9247-4876

Universidade Federal de Campina Grande, Brasil

E-mail: ingrid.paloma@estudante.ufcg.edu.br

Francisca Moisés de Sousa

ORCID: https://orcid.org/0000-0001-6152-480X

Universidade Federal de Campina Grande, Brasil

E-mail: fran_moyses@hotmail.com

Sandra Regina Dantas Baía

ORCID: https://orcid.org/0000-0002-0777-176X Universidade Federal de Campina Grande, Brasil

E-mail: sandra_reginabaia@hotmail.com

Mylena Olga Pessoa Melo

ORCID: https://orcid.org/0000-0003-4007-3063 Universidade Federal de Campina Grande, Brasil

E-mail: mylenaopm@gmail.com

Larissa Monique de Sousa Rodrigues

ORCID: https://orcid.org/0000-0002-1134-6336

Universidade Federal de Campina Grande, Brasil

E-mail: larissamonique@gmail.com

\begin{abstract}
Resumo
O objetivo desta pesquisa foi a elaboração e a caracterização físico-química de queijo petit suisse a partir de leite de búfala fermentado com kefir, utilizando-se inulina como substância prebiótica nas concentrações de 5\% (F1), 10\% (F2) e 15\% (F3). Inicialmente, obteve-se a massa do queijo quark através da fermentação do leite de búfala pelo kefir, após dessorada e lavada, a massa foi processada com os demais ingredientes até total homogeneização. A caracterização físico-química foi realizada mediante a determinação dos teores de umidade, sólidos totais, cinzas, acidez total titulável em ácido lático, $\mathrm{pH}$, açúcares redutores, não redutores e totais, e lipídeos. Os dados foram submetidos à análise de variância e a comparação de médias foi feita pelo teste de Tukey a 5\% de probabilidade. Verificou-se que a introdução de diferentes concentrações de inulina na formulação dos queijos não influenciou nos teores de cinzas, acidez titulável, pH e lipídeos, mas alterou os teores de umidade, sólidos totais e açúcares. De acordo com os resultados em geral, conclui-se que a Formulação 1, com 5\% de inulina, é a mais indicada para o processamento do queijo petit suisse prebiótico de leite de búfala, devido a utilização de menor quantidade de matéria-prima prebiótica entre as amostras, que influencia diretamente no custo do produto final e, ainda assim, mantém a funcionalidade de um alimento prebiótico conforme a legislação vigente.
\end{abstract}

Palavras-chave: Laticínios; Alimentos funcionais; Prebiótico; Bubalus bubalis; Kefir.

\section{Abstract}

The objective of this research was the elaboration and physicochemical characterization of petit suisse cheese from buffalo milk fermented with kefir, using inulin as a prebiotic substance in concentrations of 5\% (F1), 10\% (F2) and 15 $\%$ (F3). Initially, the quark cheese mass was obtained by fermenting buffalo milk by kefir, after being drained and washed, the mass was processed with the other ingredients until completely homogenized. The physicochemical characterization was carried out by determining the moisture content, total solids, ash, total acidity titratable in lactic 
acid, $\mathrm{pH}$, reducing, non-reducing and total sugars, and lipids. The data were subjected to analysis of variance and the comparison of averages was performed by the Tukey test at 5\% probability. It was found that the introduction of different concentrations of inulin in the cheese formulation did not influence the ash content, titratable acidity, $\mathrm{pH}$ and lipids, but altered the moisture content, total solids and sugars. According to the results in general, it is concluded that formulation 1, with 5\% inulin, is the most suitable for the processing of buffalo milk prebiotic petit suisse cheese, due to the use of less prebiotic raw material between samples, which directly influences the cost of the final product and still maintains the functionality of a prebiotic food in accordance with current legislation.

Keywords: Dairy products; Functional foods; Prebiotic; Bubalus bubalis; Kefir.

\section{Resumen}

El objetivo de esta investigación fue la elaboración y caracterización fisicoquímica de queso petit suisse a partir de leche de búfala fermentada con kéfir, utilizando inulina como sustancia prebiótica en concentraciones de 5\% (F1), $10 \%(\mathrm{~F} 2)$ y $15 \%$ (F3). Inicialmente, la masa de queso quark se obtenía fermentando leche de búfala por kéfir, luego de ser escurrida y lavada, la masa se procesaba con los demás ingredientes hasta homogeneizar completamente. La caracterización físico-química se realizó mediante la determinación del contenido de humedad, sólidos totales, cenizas, acidez total titulable en ácido láctico, $\mathrm{pH}$, azúcares reductores, no reductores y totales, y lípidos. Los datos se sometieron a análisis de varianza y la comparación de medias se realizó mediante la prueba de Tukey al 5\% de probabilidad. Se encontró que la introducción de diferentes concentraciones de inulina en la formulación del queso no influyó en el contenido de cenizas, acidez titulable, pH y lípidos, pero alteró el contenido de humedad, sólidos totales y azúcares. De acuerdo con los resultados en general, se concluye que la Formulación 1, con 5\% de inulina, es la más adecuada para el procesamiento de queso petit suisse prebiótico de leche de búfala, debido al uso de materia prima menos prebiótica. entre muestras, lo que influye directamente en el coste del producto final y aún mantiene la funcionalidad de un alimento prebiótico de acuerdo con la legislación vigente.

Palabras clave: Productos lácteos; Alimentos funcionales; Prebiótico; Bubalus bubalis; Kefir.

\section{Introdução}

O consumo de alimentos lácteos é significativo em todo o mundo, com uma crescente tendência de mercado nos últimos anos devido ao maior interesse dos consumidores em fermentados, que oferecem benefícios nutricionais e funcionais ao organismo humano em razão do processo de fermentação promover o aumento da biodisponibilidade de nutrientes e a redução de fatores antinutricionais pela ação de cepas específicas de bactérias ácido-láticas (García-Burgos et al., 2020).

A oferta de alimentos funcionais no mercado é ampla, mas ainda é necessário investimento em pesquisas nesta área, para que novos produtos sejam desenvolvidos e disponibilizados para beneficiar a população. Nesta perspectiva, a atuação da indústria de alimentos e de instituições de pesquisa é fundamental no desenvolvimento de tecnologias e inovações, com base no comportamento e nas expectativas dos consumidores (Filbido, Siquieri \& Bacarji, 2019).

A ingestão de alimentos funcionais pode contribuir de maneira positiva no combate a doenças crônicas, devido aos benefícios que são capazes de promover à saúde, a exemplo dos probióticos e prebióticos que estão presentes em diversos produtos neste segmento, os quais são desenvolvidos através do incremento de ingredientes capazes de agregar tais funcionalidades ao produto final, oferecendo ao consumidor benefícios como a capacidade de melhorar a saúde intestinal e o sistema imunológico, prevenir a carcinogênese, melhorar a saúde cardiovascular e promover benefícios ao metabolismo da glicose e dos lipídeos (Guimarães et al., 2019).

O kefir é um alimento potencialmente probiótico proveniente da fermentação do leite animal por grãos de kefir, que consistem em uma mistura complexa de bactérias e leveduras constituída de uma microflora mista (Yilmaz-Ersan et al., 2018). Há um crescente interesse comercial pelo kefir como uma matriz alimentar adequada para suplementação com bactérias promotoras da saúde, devido ao fornecimento de microrganismos probióticos, além disso a sua produção é realizada através de um processo simples que pode ser executado de maneira caseira (Prado et al., 2015).

A inulina, uma oligofrutose conhecida internacionalmente pelo seu potencial funcional e tecnológico, vem sendo bastante utilizada através de sua adição em alimentos devido às suas funções prebióticas (Beltrao et al., 2017). Sua influência é mínima no sabor dos produtos aos quais é incrementada como ingrediente, e atua como um excelente substituto da gordura por 
possuir capacidade de retenção de água e capacidade de geleificação; além de apresentar baixo valor calórico e altos teores de fibras solúveis (Costa et al., 2016).

O leite de búfala detém a segunda maior produção mundial e compõe uma excelente fonte de nutrientes, destacandose devido ao seu alto conteúdo de gordura, lactose, caseína, proteínas de soro de leite e minerais (Alves et al., 2019). Tradicionalmente, é conhecido devido à sua aplicação na produção de queijo mussarela, havendo uma tendência positiva do setor leiteiro de búfalos devido à diversificação do seu uso no desenvolvimento de novos produtos (Berlese, Corazzin \& Bovolenta, 2019).

O queijo petit suisse é um queijo fresco que geralmente é consumido como sobremesa no Brasil bastante apreciado principalmente pelo público infantil, podendo ser elaborado com leites provenientes de vaca, cabra, ovelha ou búfala (Vieira et al., 2014). Por ser um queijo cremoso, o desenvolvimento de um petit suisse a partir do leite de búfala se torna uma alternativa interessante, devido à maior contribuição da matéria-prima na textura final do produto, visto o seu alto percentual de gordura; $\mathrm{e}$ utilizando a combinação do kefir como cultura starter na fermentação do leite para coagulação e obtenção do queijo, e da inulina como agente prebiótico, será possível desenvolver um alimento com grande potencial funcional e tecnológico.

O objetivo do presente trabalho foi a obtenção e a caracterização físico-química de queijo petit suisse a partir de leite de búfala fermentado com kefir, utilizando-se inulina em diferentes concentrações como substância prebiótica.

\section{Metodologia}

O estudo foi desenvolvido no Laboratório de Engenharia de Alimentos (LEA), da Universidade Federal de Campina Grande (UFCG). Trata-se de uma pesquisa quantitativa por se tratar da coleta de dados numéricos através de medições de grandezas em suas respectivas unidades, utilizando-se metodologias específicas (Pereira et al., 2018).

O leite de búfala foi proveniente de uma criação de búfalas da raça Murrah (Bubalus bubalis) pertencente ao Sr. André Brasil, situada na cidade de Alagoa Nova - PB. O creme de leite foi obtido através do desnate do leite búfala em laboratório com a utilização de uma desnatadeira da marca Casa das desnatadeiras, modelo 18GR, e os demais ingredientes foram obtidos no mercado local da cidade.

Antes do processamento, a qualidade do leite de búfala foi atestada através dos testes de plataforma realizados na COAPECAL - Cooperativa Agropecuária do Cariri.

A obtenção do kefir foi realizada através da fermentação do leite de búfala desnatado, em recipiente de vidro esterilizado, utilizando-se a proporção de $15 \mathrm{~g}$ de grãos de kefir para $500 \mathrm{~mL}$ de leite, durante 24 horas em temperatura ambiente. Após o período de fermentação, o leite fermentado foi peneirado para separação dos grãos de kefir, e submetido à refrigeração a $4{ }^{\circ} \mathrm{C}$.

Para o processamento do queijo quark, o leite de búfala previamente pasteurizado e desnatado foi aquecido até $35^{\circ} \mathrm{C}$, adicionado primeiramente de 5\% de leite fermentado kefir, submetido à agitação durante dois minutos, e em seguida adicionado de $0,013 \%$ de coalho previamente diluído em água filtrada e fervida (conforme instruções do fabricante), sob agitação. Posteriormente, foi realizada a incubação a $35^{\circ} \mathrm{C}$, em estufa bacteriológica, durante o período de fermentação de 24 horas. Ao final da fermentação, a coalhada foi cortada e agitada para separação do soro, e por último foi realizada a dessoragem da massa de queijo quark através da drenagem do soro pela força da gravidade, com a suspensão da coalhada em saco de algodão esterilizado, sob refrigeração, durante 24 horas.

As matérias-primas utilizadas na elaboração das formulações do petit suisse foram: queijo quark (420 g), creme de leite (87,5 g), açúcar cristal (63,7 g), preparo de frutas sabor salada de frutas (126 g), goma xantana (2,8 g), aroma (2,8 g), sorbato de potássio $(0,35 \mathrm{~g})$ e a inulina nas concentrações $5 \%, 10 \%$ e $15 \%$ da massa total.

A elaboração do petit suisse foi realizada da seguinte forma: à massa base de queijo quark, previamente lavada com 
água potável gelada, foram adicionados o creme de leite e o açúcar, previamente pasteurizados e resfriados a $10{ }^{\circ} \mathrm{C}$, seguindo com a mistura em processador até obtenção de uma massa homogênea. Em seguida, adicionou-se o preparo de salada de frutas, a goma xantana e a inulina, procedendo-se com a mistura em processador até homogeneização completa do produto. Os queijos prontos foram acondicionados em recipientes plásticos e armazenados sob temperatura de $4^{\circ} \mathrm{C}$.

Foi realizada a caracterização físico-química do produto quanto aos parâmetros de umidade, sólidos totais, cinzas, acidez total titulável em ácido lático, $\mathrm{pH}$, açúcares redutores, não redutores e totais, de acordo com os métodos descritos por Instituto Adolfo Lutz (IAL, 2008); e lipídeos conforme método descrito por Bligh e Dyer (1959).

$\mathrm{O}$ delineamento experimental foi o de blocos inteiramente casualizados com três tratamentos ( $\mathrm{F} 1=5 \%$ de inulina, $\mathrm{F} 2$ $=10 \%$ de inulina e F3 $=15 \%$ de inulilna) e três repetições, utilizando-se o software Assistat versão 7.7 beta. Os dados foram submetidos à análise de variância (ANOVA) e a comparação de médias foi feita pelo teste de Tukey a 5\% de probabilidade.

\section{Resultados e Discussão}

As médias obtidas na caracterização físico-química do petit suisse de búfala encontram-se dispostos na Tabela 1.

Tabela 1 - Médias dos parâmetros físico-químicos das formulações do petit suisse de búfala prebiótico.

\begin{tabular}{lcccc}
\hline \multicolumn{1}{c}{ Parâmetro } & \multicolumn{3}{c}{ Formulações } & DMS \\
\cline { 2 - 4 } & F1 & F2 & F3 & \\
Umidade (\%) & $50,84 \pm 0,16 \mathrm{c}$ & $52,53 \pm 0,23 \mathrm{~b}$ & $55,20 \pm 0,13 \mathrm{a}$ & 0,4549 \\
Sólidos totais (\%) & $49,15 \pm 0,16 \mathrm{a}$ & $47,46 \pm 0,23 \mathrm{~b}$ & $44,79 \pm 0,13 \mathrm{c}$ & 0,4549 \\
Cinzas (\%) & $0,55 \pm 0,015 \mathrm{a}$ & $0,56 \pm 0,015 \mathrm{a}$ & $0,56 \pm 0,015 \mathrm{a}$ & 0,0382 \\
Acidez Total Titulável (\%) & $1,27 \pm 0,02 \mathrm{a}$ & $1,19 \pm 0,05 \mathrm{a}$ & $1,22 \pm 0,03 \mathrm{a}$ & 0,0952 \\
pH & $5,07 \pm 0,002 \mathrm{a}$ & $5,06 \pm 0,002 \mathrm{a}$ & $5,06 \pm 0,001 \mathrm{a}$ & 0,0046 \\
Açúcares redutores (\%) & $23,50 \pm 0,20 \mathrm{a}$ & $23,87 \pm 0,14 \mathrm{a}$ & $23,06 \pm 0,11 \mathrm{~b}$ & 0,3953 \\
Açúcares não redutores (\%) & $4,25 \pm 0,19 \mathrm{~b}$ & $3,81 \pm 0,40 \mathrm{~b}$ & $6,46 \pm 0,62 \mathrm{a}$ & 1,1116 \\
Açúcares totais (\%) & $27,99 \pm 0,24 \mathrm{~b}$ & $27,89 \pm 0,28 \mathrm{~b}$ & $29,87 \pm 0,70 \mathrm{a}$ & 1,1528 \\
Lipídeos (\%) & $12,9 \pm 0,02 \mathrm{a}$ & $12,9 \pm 0,05 \mathrm{a}$ & $12,8 \pm 0,12 \mathrm{a}$ & 0,2016 \\
\hline
\end{tabular}

$\mathrm{F} 1=5 \%$ de inulina, F2 = 10\% de inulina, F3 = 15\% de inulina; DMS = Desvio Médio Significativo; Médias seguidas pela mesma letra, na linha, não diferem estatisticamente entre si pelo teste de Tukey a $5 \%$ de probabilidade.

Fonte: Autores (2021).

Todas as amostras apresentaram diferença significativa a 5\% de probabilidade, pelo Teste de Tukey quanto ao teor de umidade, de maneira que as três formulações obtiveram médias acima de 50\%, com variação entre 50,84 e 55,20\%. Teores de umidade dentro desta faixa foram obtidos por Matiello et al. (2016), ao caracterizarem petit suisse de leite de ovelha com teor reduzido de lactose e diferentes concentrações de gordura e estabilizantes, em que as médias variaram entre 53,12 e 54,59\%.

Observou-se um aumento gradativo das médias de umidade à medida que as porcentagens de inulina foram elevadas. Costa et al. (2016) encontrou comportamento semelhante ao analisar petit suisse adicionado de inulina. De acordo com Pinto e Paiva (2010), a inulina é caracterizada por sua alta higroscopicidade devido à facilidade que tem de se ligar com a água, deste modo há redução na capacidade de remoção de água do produto.

O teor de sólidos totais apresentou comportamento inverso em relação ao teor de umidade, havendo diferença significativa entre as amostras, com médias mínima e máxima de 44,79 e 49,15\% para as amostras F3 e F1, respectivamente. Valores inferiores foram encontrados por Oliveira et al. (2013), para petit suisse padrão (36,98\%) e adicionado de farinha de 
chia (37,71); e por Vargas et al. (2017) ao analisarem queijo petit suisse probiótico adicionado de chia, com médias que variaram entre 32,5 e 35,15\%. Esta diferença em relação aos resultados obtidos por outros autores está associada às diferentes proporções dos ingredientes líquidos e secos, determinadas nas formulações empregadas por cada autor para o processamento do produto.

Não foi encontrada diferença significativa entre as médias de cinzas, indicando que o aumento das concentrações de inulina não provocou alterações no produto. As médias obtidas foram próximas à faixa encontrada por Sousa (2014), ao analisar queijo petit suisse de kefir sabor goiaba com inulina (0,57 a 0,65\%); e por Costa et al. (2016) ao analisarem queijo petit suisse padrão $(0,61 \%)$ e adicionado de inulina $(0,57 \%)$.

As médias de $\mathrm{pH}$ e acidez total titulável em ácido láctico não demonstraram diferença significativa entre as amostras, mostrando que o aumento das concentrações de inulina não alterou estes parâmetros. As médias de pH das três formulações encontram-se em torno de 5,0, corroborando com os baixos teores de ácido láctico encontrados nas formulações, entre a faixa de 1,19 e 1,27\%, atestando que o produto em questão é um alimento de baixa acidez. Sousa e Rapacci (2010) não observaram variação de $\mathrm{pH}$ e acidez em queijo petit suisse simbiótico fabricado com leite de búfala, adicionado de diferentes concentrações do prebiótico oligofrutose.

Valores de pH próximos foram encontrados por Cardarelli (2006), com faixa entre 4,29 e 4,68, ao analisarem queijo petit suisse simbiótico; por Veiga et al. (2000), com faixa entre 4,42 e 4,52, ao analisarem amostra de queijo petit suisse de diferentes marcas comercializadas em Campinas - SP; e por Paixão et al. (2011), com faixa entre 4,47 e 4,75 ao caracterizarem queijos petit suisse comercializados na cidade de Lavras - MG.

$\mathrm{Na}$ análise de açúcares, apenas a Formulação 3 (15\% de inulina) destacou-se, apresentando diferença significativa entre as demais amostras. De acordo com os resultados observados nas Formulação 1 (5\% de inulina) e Formulação 2 (10\% de inulina), o aumento da concentração de inulina de 5 para $10 \%$ não alterou o comportamento dos teores de açúcares do produto. Através dos resultados obtidos é possível observar que houve a diminuição dos açúcares redutores em glicose e elevação dos açucares não redutores em sacarose quando utilizou-se a concentração de $15 \%$ de inulina na formulação do queijo petit suisse, o que provocou um aumento significativo no teor de açúcares totais da amostra com esta concentração de inulina. Entretanto, Silva et al. (2011), ao desenvolverem bebidas mistas à base de cajá e caju enriquecidas com diferentes prebióticos, em que a primeira formulação continha $5 \%$ de fruto

oligossacarídeo, a segunda continha 5\% de inulina e na terceira não foram adicionados prebióticos, não observaram alterações significativas nos valores de açúcares totais, redutores e não redutores.

Quanto aos teores de lipídeos, não foi observada diferença significativa entre as amostras, comprovando que a inulina não interfere na composição lipídica do produto. Comportamento semelhante foi observado pelos autores Santos et al. (2014), ao analisarem iogurte adicionado de inulina, e Fidelis et al. (2014), ao caracterizarem iogurte natural desnatado adicionado de inulina. As médias de teor de lipídeos obtidas no presente estudo (12,8 a 12,9\%) estão acima da faixa encontrada por Costa et al. (2016), em queijo petit suisse adicionado de inulina (10,63\% para a amostra padrão e 10,87\% para a amostra com $14,25 \%$ de inulina). Esta superioridade nas médias pode ser relacionada com a utilização do leite de búfala, que se destaca devido ao seu alto conteúdo de gordura. Os autores também não observaram alterações significativas a 5\% de probabilidade, pelo Teste de Tukey, no conteúdo lipídico entre as amostras. Este comportamento confere à inulina, eficiência e potencial como substituto de gordura em produtos lácteos.

\section{Conclusão}

Através da metodologia de processamento utilizada foi possível produzir queijos petit suisse prebiótico de búfala de boa qualidade e com características aceitáveis. Com as análises físico-químicas foi possível verificar que a introdução de 
diferentes concentrações de inulina na formulação dos queijos não influenciou nos teores de cinzas, acidez titulável, pH e lipídios, mas alterou os teores de umidade, sólidos totais e açúcares.

De acordo com os resultados em geral, conclui-se que a Formulação 1, com 5\% de inulina, é a mais indicada para o processamento do queijo petit suisse prebiótico de leite de búfala, devido a utilização de menor quantidade de matéria-prima prebiótica entre as amostras, que influencia diretamente no custo do produto final e, ainda assim, mantém a funcionalidade de um alimento prebiótico conforme a legislação vigente.

Como sugestão para trabalhos futuros, podem ser realizadas análises microbiológicas voltadas para a avaliação do potencial probiótico e também para o controle sanitário do produto, além de avaliação sensorial para o estudo da preferência e da intenção de compra dos consumidores.

\section{Referências}

Alves, E. C., Soares, B. B., Almeida Neto, J. A. de, \& Rodrigues, L. B. (2019). Strategies for reducing the environmental impacts of organic mozzarella cheese production. Journal Of Cleaner Production, 223, 226-237.

Berlese, M., Corazzin., M., \& Bovolenta, S. (2019). Environmental sustainability assessment of buffalo mozzarella cheese production chain: a scenario analysis: A scenario analysis. Journal Of Cleaner Production, 238, 1-9.

Beltrao, F. A. S., Moura, C. V. R. de, Madruga, M. S., \& Andrade, A. E. B. de, (2017). Avaliação do perfil de ácidos graxos de queijo tipo chevrotin simbiótico. Rev. Inst. Laticínios Cândido Tostes, 72 (1) 11-18.

Bligh, E. G., \& Dyer, W. (1959). A rapid method of total lipid extraction and purfication. Canadian Journal of Blochemistry and Physlology, 7 (8), $911-917$.

Cardarelli, H. R. (2006). Desenvolvimento de queijo petit-suisse simbiótico. 2006. Tese de doutorado, Universidade de São Paulo, São Paulo, SP, Brasil.

Costa, E. C., Romeiro, M. M., Candido, C. J., Santos, E. F. dos, \& Novello, D. (2016). Queijo Petit Suisse com adição de inulina: análise físico-química e sensorial entre crianças. Uniabeu, 9 (22), $254-267$.

Fidelis, J. C. F., Scapim, M. R. S., Tonon, L. A. C. T., Pozza, M. S. S., Pieretti, G. G., Antigo, J. L. D., \& Madrona, G. S. (2014). Iogurte natural desnatado adicionado de inulina. Revista Brasileira de Tecnologia Agroindustrial, 8 (2), 1478 - 1487.

Filbido, G. S., Siquieri, J. P. A., \& Bacarji, A. G. (2019). Perfil do consumidor de alimentos lácteos funcionais em Cuiabá-MT. Revista Principia, (45), 31 39.

García-Burgos, M., Moreno-Fernández, J., Alférez, M. J.M., Díaz-Castro, J., \& López-Aliaga, I. (2020). New perspectives in fermented dairy products and their health relevance. Journal Of Functional Foods, 72, 1-11.

Guimarães, J. T., Balthazar, C. F., Scudino, H., Pimentel, T. C., Esmerino, E. A., Ashokkumar, M., Freitas, M. Q., \& Cruz, A. G. (2019). High-intensity ultrasound: A novel technology for the developmente of probiotic and prebiotic dairy products. Ultrasonic Sonochemistry, 57, 12-21.

Instituto Adolfo Lutz (2008). Métodos físico-químicos para análise de alimentos. IAL, $1020 \mathrm{p}$.

Matiello, E. R., Bueno, P. F., Badia, V., Polmann, G., Rigo, E., \& Bagatini, L. (2016). Caracterização sensorial e físico-química do queijo tipo petit suisse elaborado a base de leite de ovelha com teor reduzido de lactose e diferentes concentrações de gordura e estabilizantes. Revista do Congresso Sul Brasileiro de Engenharia de Alimentos, 2 (1), 1 - 10.

Oliveira, A. C. de, Deola, A. R., \& Elias R. P. (2013). Elaboração de petit suisse sabor morango adicionado de fibras e probiótico. Trabalho de conclusão de curso, Universidade Tecnológica Federal do Paraná. Medianeira, Paraná, Brasil.

Paixão, M. G., Ribeiro, O. A. S., Fonseca, R. L., Resende, C. P. A., Pinto, S. M., \& Abreu, L. R. (2011). Caracterização fisico-química de queijos petit suisse comercializados na região de Lavras - MG e adequação dos rótulos quanto a legislação. Journal of Candido Tostes Dairy Institute, 66 (383), 5 - 12.

Pereira A. S. et al. (2018). Metodologia da pesquisa científica. UAB/NTE/UFSM.

Pinto, A. L. D., \& Paiva, C. L. (2010). Desenvolvimento de uma massa funcional pronta para tortas utilizando o método de Desdobramento da Função Qualidade (QFD). Ciência e Tecnologia de Alimentos. Campinas, 30 (1), 36-43.

Prado, M. R., Blandón, L. M., Vandenberghe, L. P. S., Rodrigues, C., Castro, G. R., Thomaz-Soccol, V., \& Soccol, C. R. (2015). Milk kefir: composition, microbial cultures, biological activities, and related products: composition, microbial cultures, biological activities, and related products. Frontiers In Microbiology, 6, 1-10.

Santos, K. A., Santos, E. F., Manhani, M. R., Sanches, F. L. F. Z., Ballard, C. R., \& Novello, D. (2014). Avaliação das características sensoriais e físicoquímicas de iogurte adicionado de inulina. Revista Uniabeu, 7 (15), 50 - 65.

Silva, L. M. R., Lima, A. S., Maia, G. A., Rodrigues, M. C. P., Figueiredo, R. W., \& Sousa, P. H. M. (2011). Desenvolvimento de bebidas mistas à base de cajá (Spondias mombin l.) E caju (Anacardium occidentale) enriquecidas com frutooligossacarídeos e inulina. Archivos Latinoamericanos de Nutrición, 61 (2). 
Research, Society and Development, v. 10, n. 1, e37810111856, 2021

(CC BY 4.0) | ISSN 2525-3409 | DOI: http://dx.doi.org/10.33448/rsd-v10i1.11856

Sousa, P.B. (2014). Queijo petit-suisse de kefir sabor goiaba com inulina: elaboração e avaliação físico-química, microbiológica e sensorial. Dissertação de Mestrado, Instituto Federal de Educação, Ciência e Tecnologia do Ceará, Limoeiro do Norte, Ceará, Brasil.

Sousa, L. N., \& Rapacci, M. (2010). Queijo petit suisse simbiótico fabricado com leite de búfala. XIII Seminário de Iniciação Científica da Pontfícia Universidade Católica do Paraná, Curitiba.

Vargas, P. O., Correa, K. P., Condé, J. L., Martins, A. D. O., \& Silva, F. J. M. (2017). Desenvolvimento de queijo petit suisse probiótico adicionado de farinha de chia. Brazilian Journal Of Food Research, 8 (3), 74 - 87.

Vieira, A. D. S., Santos, K. M. O., Barcelos, S. C. de, Oliveira, I. C., \& Saad, S. M. I. (2014). Processamento artesanal de queijo caprino simbiótico tipo petit suisse. Comunicado Técnico, n.114, Embrapa, Sobral, Ceará, Brasil.

Yilmaz-Ersan, L., Ozcan, T., Akpınar, A., \& Șahin, S. (2018). Comparison of antioxidant capacity of cow and ewe milk kefirs. Journal Of Dairy Science, 101 (5), 3788-3798. 